A. Pramesh Rao, G. Swarup and Gopal-Krishna, eds.

\title{
Multifrequency GMRT Observations of HII Regions
}

\author{
A. Omar \\ Raman Research Institute, Bangalore, India \\ J.N. Chengalur and D.A. Roshi \\ National Centre for Radio Astrophysics, TIFR, Pune, India
}

\begin{abstract}
The electron temperatures of the compact cores of the galactic HII regions S206 and S209 have been determined by radio continuum observations near 235, 327 and $610 \mathrm{MHz}$ using the Giant Meterwave Radio Telescope (GMRT). The resolution of our maps are 11" and 6" at 327 and $610 \mathrm{MHz}$ respectively. These are the highest resolution low frequency maps of these HII regions.
\end{abstract}

\section{Introduction}

The optical depth for free-free emission from HII regions varies as $\tau \sim \nu^{-2.1}$. At sufficiently low frequencies therefore these regions become optically thick and the observed brightness temperature $T_{B}$ is equal to the electron temperature $T_{e}$.

Accurate measurement of $T_{e}$ however requires high resolution images, particularly so for HII regions with low emission measure (EM) where often only a very compact core is optically thick even at frequencies as low as a few hundred MHz. We present here preliminary analysis of GMRT low frequency $(232,327$ and $610 \mathrm{MHz}$ ) maps of the HII regions S206 and S209.

\section{Observations \& Discussion}

At $610 \mathrm{MHz}$ correction for the differing sky background at the flux calibrator and the source was made by actually measuring the system temperature at these two locations. The $327 \mathrm{MHz}$ maps were scaled to give the same flux for point sources as the WENSS survey, and at $232 \mathrm{MHz}$ the flux scale was set to that of the Miyun survey. Both HII regions show structures on scales of a few arcseconds including shells, rings and cometary type structures, enveloped by a diffuse emission. Here we restrict ourselves to a discussion of the electron temperature in the bright 'core' regions. A more detailed ananlysis will be presented in Omar et al. 2000.

For a homogeneous, spherically symmetric HII region (which we approximate the core region to) we have the flux $S$ given by (Hjellming et al. 1969, Mezger et al. 1967)

$$
S=3.07 \times 10^{-2} T_{e} \nu^{2} \Omega\left(1-e^{-\tau(\nu)}\right) ; \tau(\nu)=1.643 \times 10^{5} \nu^{-2.1} E M T_{e}^{-1.35}
$$




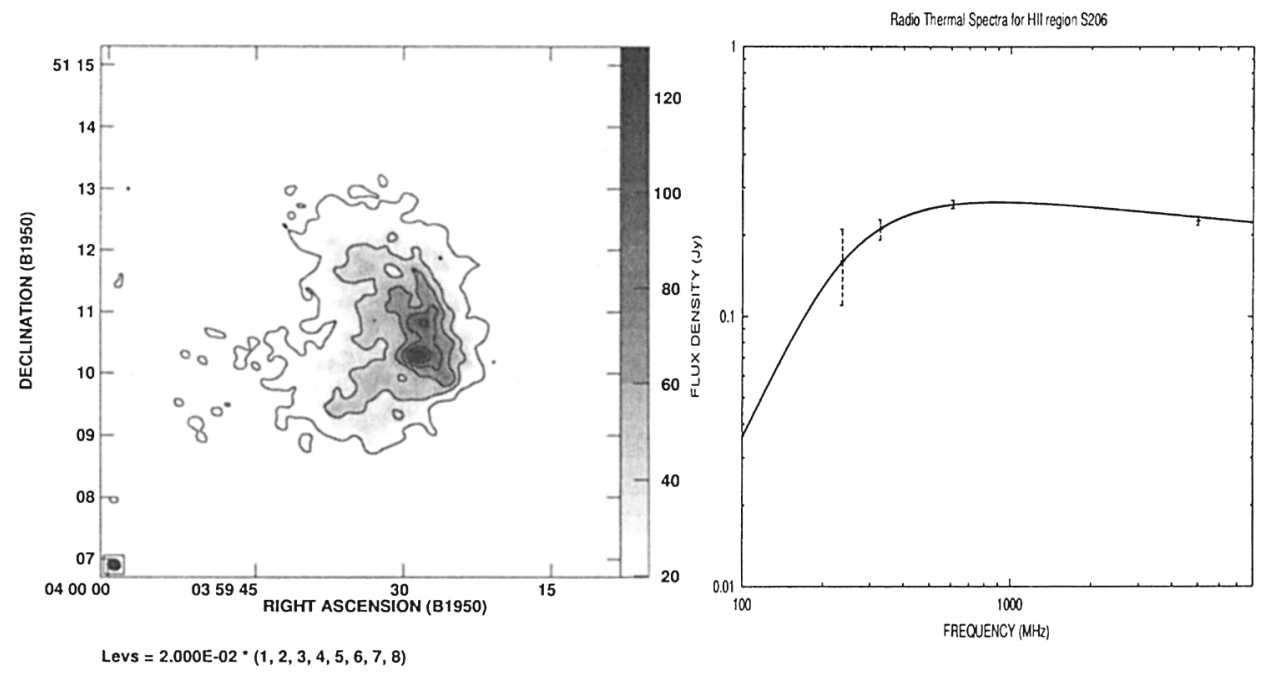

Figure 1. [A]610 MHz GMRT image of S206. [B]Measured flux for S206 plotted on an isothermal nebula model.

We can model the HII region by solving these equations iteratively for different $E M$ and $T_{e}$ using observations at a minimum of two frequencies (eg. Terzian et al. 1968). For the core region in S206, the temperature we determine from our 235,327 and $610 \mathrm{MHz}$ fluxes is $10500 \pm 1000^{\circ} \mathrm{K}$ and the emission measure is $2.5 \times 10^{5} \mathrm{~cm}^{-6}$ pc. The predicted flux at $4.9 \mathrm{GHz}$ using these numbers is in good agreement with the WSRT measurement by Deharveng et al.(1976). This temperature is also in reasonable agreement with the value of $8400 \pm 800^{\circ} \mathrm{K}$ obtained using the H94 $\alpha$ recombination line by Carral et al.(1981). The temperature we obtain for core region of S209 is $7200 \pm 700^{\circ} \mathrm{K}$ again in reasonable agreement with the value of $8280 \pm 800^{\circ} \mathrm{K}$ obtained using the $\mathrm{H} 137 \beta$ recombination line by Churchwell et al. (1978).

\section{References}

Carral et al. 1981, A\&A, 95, 388

Churchwell et al. 1978, A\&A, 70, 719

Deharveng et al. 1976 A\&A, 48, 63

Hjellming et al. 1969, ApJ, 157, 573

Mezger et al. 1967, ApJ, 147, 471

Omar et al. 2000, in preparation

Terzian et al. 1968, Ap. L., 1, 153 